\title{
Müllerian Adenosarcoma of the Urinary Bladder: Clinicopathologic and Immunohistochemical Features with Novel Genetic Aberrations
}

Joseph Sanfrancesco ${ }^{1}$, Sean R Williamson ${ }^{3,4,5}$, Jennifer B. Kum ${ }^{1}$, Shaobo Zhang ${ }^{1}$, Mingsheng Wang ${ }^{1}$, Antonio Lopez-Beltran ${ }^{6}$, Rodolfo Montironi ${ }^{7}$, Thomas A. Gardner ${ }^{2}$, Liang Cheng ${ }^{1,2}$

From the Departments of Pathology ${ }^{1}$ and Urology ${ }^{2}$, Indiana University School of Medicine, Indianapolis, Indiana; ${ }^{3}$ Department of Pathology and Laboratory Medicine and ${ }^{4}$ Josephine Ford Cancer Institute, Henry Ford Health System, Detroit, MI, United States;

${ }^{5}$ Department of Pathology, Wayne State University School of Medicine, Detroit, MI, United States; ${ }^{6}$ Unit of Anatomical Pathology, Department of Surgery, Faculty of Medicine, Cordoba, Spain and Champalimaud Clinical Center, Lisbon, Portugal;

${ }^{7}$ Department of Pathological Anatomy and Histopathology, School of Medicine, Polytechnic University of the Marche Region (Ancona), Ancona, Italy.

Key Words: Urinary bladder, adenosarcoma, molecular genetics/cytogenetics, endometriosis, differential diagnosis.

Total number of text pages, 20; Number of tables, 0 ; Number of figures, 4 .

Conflict of Interest: None.

Address correspondence and reprint requests to Liang Cheng, M.D., Department of Pathology and Laboratory Medicine, Indiana University School of Medicine, 350 West 11 th Street, IUHPL Room 4010, Indianapolis, IN 46202, USA. Telephone: 317-4916442; Fax: 317-491-6419; E-mail: liang_cheng@yahoo.com

This is the author's manuscript of the article published in final edited form as: Sanfrancesco, J., Williamson, S. R., Kum, J. B., Zhang, S., Wang, M., Lopez-Beltran, A., ... Cheng, L. (2017). Müllerian Adenosarcoma of the Urinary Bladder: Clinicopathologic and Immunohistochemical Features with Novel Genetic Aberrations. Clinical Genitourinary Cancer. https://doi.org/10.1016/j.clgc.2017.05.020 


\section{Conflict of Interest: None.}

\section{MicroAbstract}

We reported the first case of Müllerian adenosarcoma arising in the urinary bladder with extensive clinicopathologic, immunohistochemical, and molecular analyses. Given the tendency of low-grade müllerian adenosarcomas to recur and/or develop stromal overgrowth, discerning this diagnostic entity from benign extra-uterine processes (such as endometriosis) has important diagnostic and clinical implications. 


\begin{abstract}
Müllerian adenosarcoma is a biphasic neoplasm most commonly of the uterus and less frequently of the ovary. It has been rarely described to occur in other sites such as peritoneum and liver. In this study, we report the clinicopathologic, immunohistochemical and molecular features of a primary müllerian adenosarcoma of the urinary bladder in a 62-year-old woman. To our knowledge, this is the first report of muillerian adenosarcoma primary to the urinary bladder in the literature. Light microscopy showed a biphasic epithelial and stromal tumor with benign-appearing glands surrounded by endometrial-type stroma that is densely cellular with increased mitotic figures. The stroma surrounding the glands is more cellular than the intervening areas, which are more loose and edematous. Immunohistochemistry profile included positive staining for Pax $2 / 8$ within the glands, for CD10 and WT- 1 within the spindled stroma, and for estrogen and progesterone receptors in both. Staining for desmin, GATA3, p63, and human papilloma virus (HPV) is negative. Molecular analyses identified mutations in AKT1 E17K, FLT3 D835N, KRAS G12D and HRAS G12S. These novel molecular aberrations have yet to be reported in the medical literature. X chromosome inactivation analysis revealed a clonal pattern in the stromal component and a non-clonal pattern in the epithelial component. Currently, the patient is disease/recurrence-free after regular follow-up of approximately 84 months. This case represents the first reported diagnosis of müllerian adenosarcoma arising in the urinary bladder with extensive clinicopathologic, immunohistochemical, and molecular analyses.
\end{abstract}

(Word counts:239) 


\section{Introduction}

Müllerian (mesodermal) adenosarcoma is a distinctive type of mixed epithelial and mesenchymal tumor of the female genital tract that was first described in $1974 .^{1}$ These tumors are most often found in the uterus. ${ }^{1-3}$ The ovary is the most common extrauterine site. ${ }^{4}$ These tumors have also been found in the pelvis, ${ }^{5-7}$ vagina, ${ }^{8}$ and liver. ${ }^{9}$ The epithelial component is typically composed of benign-appearing glands that can be dilated or slit-like. The epithelium is most often endometrioid, but can also be mucinous (endocervical), ciliated, squamous, or serous. ${ }^{2}$ Occasionally, tumors with an atypical glandular component have been described. ${ }^{2}$ The mesenchymal component usually consists of a low-grade malignant stroma may exhibit overgrowth, sometimes significant, compared to the epithelial component. Tumors with sarcomatous overgrowth and aggressive clinical course have been described. ${ }^{1}$ There are 13 reported cases of müllerian adenosarcoma occurring in patients being treated with Tamoxifen therapy. ${ }^{10-15}$

We report a case of müllerian adenosarcoma of the urinary bladder occurring in a patient with long-standing history of endometriosis. In addition to an extensive immunohistochemical panel, we performed next generation sequencing, virtual karyotype analysis, and $\mathrm{X}$-chromosome inactivation analysis. To our knowledge, this is the first case of müllerian adenosarcoma primary to the urinary bladder with concurrent histopathologic, immunohistochemical and molecular analyses, including novel molecular aberrations yet to be reported in the literature. 


\section{Materials and Methods}

\section{Clinical Presentation}

A 62 year-old woman presented for urological evaluation due to gross hematuria. Her previous surgical history included a hysterectomy at an outside institution 15 years prior. Pathologic findings in the uterus from that procedure were not known. Cystoscopy had been performed 12 years prior for hematuria, which showed a mass at the base of the urinary bladder clinically described as an endometrioma. This was reported to be compatible with known history of endometriosis in conjunction with a urinary tract infection at that time. Pathology from this prior resection was not available for review.

Due to recurrent hematuria, cystoscopy and subsequent transurethral resection of a $2-3 \mathrm{~cm}$ bladder mass located adjacent to the right ureteral orifice were performed, clinically believed to represent a recurrent endometrioma. Pathologic review of the specimen yielded a diagnosis of endometrial-type tissue with atypical spindle cell proliferation. Given the suspicion for a low-grade müllerian adenosarcoma and the inability to completely resect the lesion transurethrally, a partial cystectomy was performed. Intraoperatively, the lesion appeared to extend into the bladder wall and the right distal ureter.

Pathologic evaluation of the submitted specimens identified a urinary bladderbased tumor consisting of benign endometrial glands surrounded by highly cellular and atypical spindle cells consistent with a müllerian (mesodermal) adenosarcoma. Background endometriosis was also identified. Surgical margins were negative and the lesion was considered completely excised. Lymph nodes were not sampled. 
Since the repair, the patient has had no additional complications from the procedure and is disease-free to date after close observation and regularly scheduled follow-up (approximately 84 months).

\section{Immunohistochemistry and Molecular Analyses}

A search through our institution's surgical pathology files identified the prior transurethral resection of the urinary bladder and subsequent partial cystectomy. Hematoxylin and eosin as well as immunohistochemical stains (with appropriate controls) from both cases were obtained for review. Paraffin blocks were obtained for molecular studies. This review was approved by our institutional review board (IRB).

Antibodies directed against beta-catenin (M3539, dilution 1:60, Dako Corp. Carpentaria, CA), CA-125 (CMA743, Cell Marque, Darmstadt, Germany), CD10 (IR 648, Dako Corp,.), CK7 (IR 619 Dako Corp.), CK20 (IR 777, Dako Corp.), desmin (IR 606; Dako Corp.), ERG (IR 659 Dako Corp.), estrogen receptor (IR 151, Dako Corp.), GATA3 (SC 268, dilution 1:50, Santa Cruz Biotechnology, Dallas, TX), HPV (M3528, dilution 1:25, Dako Corp.), Ki-67 (IR 626, dilution 1:300, Dako Corp.), p63 (M7247, dilution 1:400, Dako Corp.), Pax-2 (Invitrogen, dilution 1:100, Thermo Fisher Scientific, Waltham, MA ), Pax-8 (EP298, Cell Marque), progesterone receptor (IR 068; Dako Corp.), smooth muscle actin (1 A4; Dako Corp), TTF-1 (IR 056, Dako Corp.), and WT-1 (IR 055, Dako Corp.) were performed in a Dako Corp. automated instrument. Positive and negative controls gave appropriate results for each antibody performed.

Molecular studies including next generation sequencing (NGS), single nucleotide polymorphism array assay and X chromosome inactivation analysis were performed. 
DNA was obtained from selected formal fixed paraffin embedded (FFPE) tissue to obtain nuclei acid extract to perform multiplexed targeted sequencing assays to detect for mutations. Regions of viable tumor were selected by an experienced genitourinary pathologist (L.C.) on hematoxylin and eosin stained slides and corresponding unstained slides were deparaffinized with two washes of xylene, 15 minutes each, and subsequently washed twice with absolute ethanol, 10 minutes each, and then air-dried in a fume hood. Subsequent protein digestion and DNA extraction were completed. Pre-screening for specimen adequacy was performed according to the protocols of TruSeq Amplicon Cancer Panel quality control kit for FFPE samples (ACP; Illumina, San Diego, CA). Once the sample was deemed adequate, the extracted DNA was analyzed via single-tube reaction evaluating 48 different genes targeted by 212 amplicons on the Illumina MiSeq system.

For virtual karyotyping, DNA was obtained from manually microdissected $10 \mu \mathrm{m}$ paraffin sections according to previously described protocol and processed with an FFPEoptimized protocol based on the GeneChip Mapping 250K Nsp SNP (single nucleotide polymorphism) Assay Kits (Affymetrix, Santa Clara, CA) as previously described ${ }^{16-18}$. $\mathrm{X}$ chromosome inactivation analysis was performed using DNA prepared from the cystectomy specimen, using methods previously described ${ }^{19-21}$. Analysis utilized a highly polymorphic CAG repeat in exon 1 of the human androgen receptor gene on chromosome Xp1 1-12. Briefly, the DNA extract was digested with HhaI (New England Biolabs Inc., Beverly, MA) and amplified in a polymerase chain reaction mixture containing $0.1 \mu \mathrm{L}$ of a $\alpha-{ }^{32} \mathrm{P}$-labeled deoxyadenosine triphosphate $(3000 \mathrm{Ci} / \mathrm{mmol} / \mathrm{L})$, AR-sense and AR-antisense primers (each at $4 \mathrm{~mol} / \mathrm{L}$ ), $4 \%$ dimethyl sulfoxide, $\mathrm{MgCl}_{2}$ at 
$2.5 \mathrm{mmol} / \mathrm{L}$, all 4 deoxynucleoside triphosphates (each at $300 \mu \mathrm{mol} / \mathrm{L}$ ), and $0.5 \mathrm{U}$ of Taq DNA polymerase (Perkin-Elmer Cetus, Foster City, CA). The samples were then heated to $95^{\circ} \mathrm{C}$ for 5 minutes and then placed on ice. Electrophoresis was performed using $6.5 \%$ denaturing polyacrylamide gel at $80 \mathrm{~W}$ for 3 hours, followed by autoradiography with Biomax MS film (Kodak Company, Rochester, NY) for 8 to 18 hours. Control tissue yielded an appropriate reaction.

\section{Results}

\section{Histopathologic Findings}

The partial cystectomy specimen consisted of a portion of urinary bladder, anterior vaginal wall, and right ureteral orifice measuring $6.0 \times 5.0 \times 3.6 \mathrm{~cm}$. A distinct mass was not appreciated; however, sectioning revealed a gray-white to red-brown cut surface remarkable for fibrosis admixed with areas of softening. These foci connect with lobulated and polypoid areas located on the mucosal surface.

The tumor was centered in the muscular wall of the urinary bladder, involving the adjacent perivesical and peri-ureter soft tissue. The tumor was characterized by benignappearing glands lined by predominantly cuboidal cells with variably eosinophilic cytoplasm. Mitotic figures and nuclear atypia were essentially being absent (Fig. 1A, B). Occasionally, glands were lined by flattened and pseudostratified epithelium. The glands were surrounded by endometrial-type stroma that was densely cellular with mild nuclear atypia and increased mitoses (up to 3 per 10 high-powered fields) [Fig. 1C, D]. The stroma surrounding the glands was more cellular than the intervening areas which was looser, edematous, and somewhat myoid-appearing (Fig. 1E). The tumor also makes 
intraglandular papillary-like projections (lined by bland cuboidal and occasionally pseudostratified cells) resembling phyllodes tumor of the breast (Fig. 1F). When compared to the prior transurethral resection, the stroma in the partial cystectomy specimen was more atypical in appearance and indeed possessed features of low-grade malignancy. There was also intravascular tumor in thin and thick walled vessels (Fig. 2A). Heterologous stromal elements and high-grade sarcomatous overgrowth were not identified. The uninvolved urinary bladder contained foci of endometriosis.

The tumor focally extended to cauterized muscularis propria in the main specimen, but it was unknown whether this area represented true margin. All additional inked and separately submitted margins were negative for tumor.

\section{Immunohistochemical Findings}

The benign glandular elements stain for Pax-2, Pax-8, and CK7 (focal, patchy) [Fig. 3A-B].Staining within the sarcomatous stroma shows positive immunoreactivity for CD10, WT-1, and smooth muscle actin (patchy) [Fig. 3C-E]. CA-125, estrogen receptor, progesterone receptor, and beta-catenin (nuclear) were positive in both components (Fig F-H). Overall, staining for CK20, desmin, GATA3, HPV, p63, and TTF-1 is negative in both components. Staining for ERG and elastin highlights tumor within lymphovascular spaces (Fig. 2B). Ki-67 shows a proliferation index of approximately $1 \%$.

\section{Mutation Analysis}

Multiplexed targeted sequencing (Next Generation Sequencing, NGS) identified the following mutations: HRAS G12S, KRAS G12D, AKT1 E17K, and FLT3 D835N. 
X chromosome Inactivation Analysis

$\mathrm{X}$-chromosome inactivation analysis revealed a clonal pattern, i.e. non-random pattern of X chromosome inactivation, in the stromal component that is characterized by a single androgen receptor band after digestion with $\mathrm{HhaI}$ restriction endonuclease. The epithelial component revealed a random pattern of $\mathrm{X}$ chromosome inactivation (Fig. 4).

Virtual Karyotyping by Single Nucleotide Polymorphism Array Assay

The specimen showed discrete chromosomal imbalances in chromosomes 1 and 3 . Chromosome 1 showed a $47.3 \mathrm{Mb}$ region of gain in the long arm: $1 \mathrm{q}(23.3-32.3$, 160285587-209409174/hg17). Chromosome 3 showed a $21.4 \mathrm{Mb}$ region of loss in the short arm: 3p(14.1-22.1, 43012181-64488564/hg17) [Fig. 4].

\section{Discussion}

Primary müllerian (mesodermal) adenosarcoma of the urinary bladder is an exceptionally rare tumor with no prior report of extensive immunohistochemical or molecular findings in the literature. In this article, we report the first case of müllerian adenosarcoma of the urinary bladder with extensive morphologic and immunophenotypic classification in addition to detection of novel molecular aberrations including AKT1, HRAS, FLT3, and KRAS somatic mutations by NGS, imbalances of chromosomes 1 and 3, as well as X chromosome inactivation.

Prior extra-gynecologic tract cases of müllerian adenosarcoma have been reported in the peritoneum, ureter, liver, prostate, testis, and seminal vesicles ${ }^{22-25}$. While the 
etiologies of these rare occurrences of müllerian adenosarcoma in men have yielded several hypotheses of their origin, the extra-uterine/ovarian cases within the female population have been almost exclusively associated with endometriosis (or müllerianosis $)^{22}$. Additionally, the association between mesodermal adenosarcoma and tamoxifen therapy is well documented; however, our patient had no reported use of $\operatorname{tamoxifen}^{10-15}$.

Histopathologically, müllerian/mesodermal adenosarcoma is a biphasic neoplasm consisting of malignant mesenchymal and benign (to mildly atypical) epithelial components ${ }^{1}$. The epithelial component typically consists of evenly distributed glands lined by endometrioid-like epithelium (cuboidal or columnar cells with occasional pseudostratification) that is not overtly malignant. The background mesenchymal stroma is variably cellular, with increased cellularity around the benign glands creating a "cuffed or collared" appearance ${ }^{4}$. The stromal cells are mild to moderately atypical with a variable amount of increased mitotic figures. The cellular stroma can extend into glandular or cystic spaces, in a papillary or polypoid fashion, imparting an appearance most analogous to phyllodes tumor of the breast ${ }^{2}$. These projections are lined by benign to mildly atypical epithelial cells. Again, similar to phyllodes tumor of the breast, müllerian adenosarcomas can undergo stromal overgrowth, with rhabdomyomatous features, and are classified as adenosarcoma with sarcomatous overgrowth according to the WHO Classification of Female Reproductive Organs ${ }^{10,26}$. Adenosarcomas are typically positive for CD10, estrogen (ER) and progesterone receptors (PR) within the stromal component as well as Pax-2/8 within the benign epithelial components. In our immunohistochemical studies, we additionally identified positive immunoreactivity in the 
stroma for WT-1, SMA (focal), beta-catenin (focal), and CA-125 (focal). Within the benign epithelial component, we identified further positive immunoreactivity for CK7 (focal), ER, PR, and beta-catenin (nuclear).

To our knowledge, this is only the third proposed case of müllerian adenosarcoma of the urinary bladder published in peer-reviewed journals ${ }^{27,28}$. The first reported case had a very limited description of morphologic features and was postulated to arise out of endometriosis in the prevesical fat rather than the urinary bladder ${ }^{27}$. Additionally, immunohistochemical or molecular studies were not performed ${ }^{27}$. The second previously reported occurrence was a single case of presumed urinary bladder adenosarcoma in 2002 , but the pathology was never specifically reviewed to confirm the diagnosis ${ }^{28}$. Limited immunohistochemical findings were described (per report, without review) and no molecular studies were performed. Findings in our case are diagnostic of müllerian adenosarcoma by morphologic, immunophenotypic, and molecular findings. The stromal features including increased mitotic activity, cellular atypia, and "cuffing" around benign glands exceed any benign process. Additionally, the phyllodes-like growth patterns in conjunction with our immunophenotypic findings are quite consistent with this diagnosis. With no stromal overgrowth identified, a classification of low-grade adenosarcoma was most appropriate.

Few studies have evaluated molecular aberrations seen in primary uterine müllerian adenosarcoma. Cytogenetic and molecular analyses identifying chromosome gains/losses and/or complex karyotype involving chromosomes such as 2, 8, 10, 13, 19 and 21 have been reported. ${ }^{29,30}$ Additionally, somatic mutations of numerous genes including MDM2, CDK4, DDIT3, CKDN2A, FGFR2 as well as alterations in the 
PIK3CA/AKT/PTEN pathway have been identified ${ }^{30-32}$. One prior study evaluated tumors by DNA flow cytometric analysis and while assessing for DNA ploidy, a subset were DNA diploid and all cases had an S-phase fraction $<10 \%{ }^{33}$.

After an extensive literature search, we have found a significant paucity of molecular/cytogenetic studies of extra-uterine mesodermal adenosarcomas. While our findings in the AKT1 pathway are similar to those previously reported in primary uterine adenosarcoma, the mutations of HRAS, KRAS, and FLT3 are unique. It should be noted that TruSeq Amplicon-Cancer Panel used to analyze this tumor (by NGS) did evaluate for various other previously described mutations and these were not identified in this tumor. Our reporting of chromosomal imbalances in chromosome 1 and 3 are the first of their kind in muillerian adenosarcoma. Of note, the $3 p$ loss region did not include the VHL gene, but it did include the PBRM1 gene. While PBRM1 has been reported to be mutated in a high percentage of clear cell renal cell carcinoma tumors, we found no prior report of this gene being lost or mutated in müllerian adenosarcomas ${ }^{34}$.

The differential diagnosis in a case such as this depends on the presumed site of origin for the lesion. Given the localization of the tumor to the urinary bladder wall, primary bladder neoplasia, neoplasia arising out of metaplasia, or a metastatic lesion from another primary site should be primary considerations. Primary sarcomatous lesions of the urinary bladder to consider include leiomyosarcoma and sarcomatoid urothelial carcinoma. While leiomyosarcoma is the most common bladder sarcoma, this tumor lacked morphologic features typically associated with the diagnosis including interlacing fascicles of cigar-shaped nuclei or significant immunoreactivity with desmin, caldesmon, or smooth muscle actin ${ }^{35}$. A diagnosis of sarcomatoid urothelial carcinoma could be 
considered; however, the lack of a malignant epithelial component, presence of benign glandular morphology, and lack of immunoreactivity with p63, GATA3, or cytokeratin (within the sarcomatous component) argued against this diagnosis ${ }^{36}$.

Previously reported mass forming lesions of the urinary bladder associated with endometriosis, or less commonly müllerianosis, include mass-forming endometriosis, clear cell adenocarcinoma, endometrioid adenocarcinoma, and endometrial stroma sarcoma $^{37-39}$. Given the stromal cellularity, infiltrative nature, increased mitotic activity, and nuclear atypia, features in the stromal component of this lesion far surpassed what is expected in a benign endometrioma ${ }^{40}$. While clear cell or endometrioid adenocarcinoma arising out of endometriosis could create a mass-forming lesion with glandular morphology, these diagnoses were not a consideration in this case as the lesion lacked a malignant epithelial component. Given the stromal predominance of these lesions, lowgrade endometrial stromal sarcoma (ESS) could be a diagnostic consideration. Tian et al previously described a case series of 6 ESSs arising out of the urinary bladder ${ }^{39}$. While the immunophenotypic overlap is intriguing, their study only described a single case arising out of the urinary bladder (the remainder were remote metastases) and furthermore, ESS in their study lacked a benign glandular component ${ }^{39}$.

Adenosarcomas have been described to recur in up to $30 \%$ of cases; however, metastasis is very uncommon and has only been reported in cases of stromal overgrowth $^{41}$. While the pathology from this patient's remote hysterectomy was unknown at the time of her current diagnosis, it is highly unlikely this represents a metastasis from a prior uterine, ovarian, or cervical adenosarcoma. Given the location being centered in the urinary bladder wall and lack of involvement of the vaginal wall, this tumor does not 
represent a vaginal primary extending into the urinary bladder. Of note, the patient's prior resection of a clinically-assessed endometrioma very well could have been an undiagnosed müllerian adenosarcoma which subsequently recurred. Unfortunately, the absence of prior pathology material makes this purely speculative.

\section{Conclusions}

Overall, extra-uterine müllerian adenosarcoma of the urinary bladder is exceedingly rare. We believe this represents the first study of extra-gynecologic müllerian (mesodermal) adenosarcoma that includes extensive immunophenotypic studies and molecular analysis with novel cytogenetic findings including mutations in KRAS, HRAS, AKT1, and FLT3. The possibility for endometriosis to form mass-like lesions in the urinary bladder is well known. Given the tendency of low-grade müllerian adenosarcomas to recur and/or develop stromal overgrowth, discerning this diagnostic entity from benign extra-uterine processes (such as endometriosis) has important diagnostic and clinical implications. 


\section{Clinical practice points:}

- Extra-uterine müllerian adenosarcoma is rare. We report the clinicopathologic, immunohistochemical and molecular features of a primary müllerian adenosarcoma of bladder.

- Immunohistochemistry profile included positive staining for PAX8 within the glands, for CD10 and WT-1 within the spindled stroma

- Molecular analyses identified mutations in AKT1 E17K, FLT3 D835N, KRAS G12D and HRAS G12S.

- X chromosome inactivation analysis revealed a clonal pattern in the stromal component and a non-clonal pattern in the epithelial component.

- Given the tendency of low-grade müllerian adenosarcomas to recur and/or develop stromal overgrowth, discerning this diagnostic entity from benign extra-uterine processes (such as endometriosis) has important diagnostic and clinical implications.

Acknowledgments: The authors would like to thank Natasha Gibson for excellent editorial assistance. We are grateful to Dr. Federico A. Monzon for experimental assistance in virtual karyotyping. 


\section{References:}

1. Clement PB, Scully RE. Mullerian adenosarcoma of the uterus. A clinicopathologic analysis of ten cases of a distinctive type of mullerian mixed tumor. Cancer 1974; $34: 1138-49$

2. Clement PB, Scully RE. Mullerian adenosarcoma of the uterus: a clinicopathologic analysis of 100 cases with a review of the literature. Hum Pathol 1990; 21:363-81

3. Clement PB. Mullerian adenosarcomas of the uterus with sarcomatous overgrowth. A clinicopathological analysis of 10 cases. Am J Surg Pathol 1989; 13:28-38

4. Eichhorn JH, Young RH, Clement PB, Scully RE. Mesodermal (mullerian) adenosarcoma of the ovary: a clinicopathologic analysis of 40 cases and a review of the literature. Am J Surg Pathol 2002; 26:1243-58

5. Clement PB, Scully RE. Extrauterine mesodermal (mullerian) adenosarcoma: a clinicopathologic analysis of five cases. Am J Clin Pathol 1978; 69:276-83

6. Patrelli TS, Silini EM, Gizzo S, et al. Extragenital Mullerian adenosarcoma with pouch of Douglas location. BMC Cancer 2011; 11:171

7. Ostor AG, Nirenberg A, Ashdown ML, Murphy DJ. Extragenital adenosarcoma arising in the pouch of Douglas. Gynecol Oncol 1994; 53:373-5

8. Judson PL, Temple AM, Fowler WC, Jr., Novotny DB, Funkhouser WK, Jr. Vaginal adenosarcoma arising from endometriosis. Gynecol Oncol 2000; 76:123-5

9. N'Senda P, Wendum D, Balladur P, et al. Adenosarcoma arising in hepatic endometriosis. Eur Radiol 2000; 10:1287-9 
10. Carvalho FM, Carvalho JP, Motta EV, Souen J. Mullerian adenosarcoma of the uterus with sarcomatous overgrowth following tamoxifen treatment for breast cancer. Rev Hosp Clin Fac Med Sao Paulo 2000; 55:17-20

11. Clement PB, Oliva E, Young RH. Mullerian adenosarcoma of the uterine corpus associated with tamoxifen therapy: a report of six cases and a review of tamoxifenassociated endometrial lesions. Int J Gynecol Pathol 1996; 15:222-9

12. Arici DS, Aker H, Yildiz E, Tasyurt A. Mullerian adenosarcoma of the uterus associated with tamoxifen therapy. Arch Gynecol Obstet 2000; 264:105-7

13. Bocklage T, Lee KR, Belinson JL. Uterine mullerian adenosarcoma following adenomyoma in a woman on tamoxifen therapy. Gynecol Oncol 1992; 44:104-9

14. Jagavkar RS, Shakespeare TP, Stevens MJ. Endometrial adenosarcoma with adjuvant tamoxifen therapy for primary breast carcinoma. Australas Radiol 1998; 42:157-8

15. Jessop FA, Roberts PF. Mullerian adenosarcoma of the uterus in association with tamoxifen therapy. Histopathology 2000; 36:91-2

16. Suzuki M, Nagura K, Igarashi H, et al. Copy number estimation algorithms and fluorescence in situ hybridization to describe copy number alterations in human tumors. Pathol Int 2009; 59:218-28

17. Kim HJ, Shen SS, Ayala AG, et al. Virtual-karyotyping with SNP microarrays in morphologically challenging renal cell neoplasms: a practical and useful diagnostic modality. Am J Surg Pathol 2009; 33:1276-86

18. Lyons-Weiler M, Hagenkord J, Sciulli C, Dhir R, Monzon FA. Optimization of the Affymetrix GeneChip Mapping 10K 2.0 Assay for routine clinical use on formalinfixed paraffin-embedded tissues. Diagn Mol Pathol 2008; 17:3-13 
19. Katona TM, Jones TD, Wang M, et al. Genetically heterogeneous and clonally unrelated metastases may arise in patients with cutaneous melanoma. Am J Surg Pathol 2007; 31:1029-37

20. Wang X, Wang M, MacLennan GT, et al. Evidence for common clonal origin of multifocal lung cancers. J Natl Cancer Inst 2009; 101:560-70

21. Sung MT, Wang M, MacLennan GT, et al. Histogenesis of sarcomatoid urothelial carcinoma of the urinary bladder: evidence for a common clonal origin with divergent differentiation. J Pathol 2007; 211:420-30

22. Fleshman RL, Wasman JK, Bodner DG, Young RH, MacLennan GT. Mesodermal adenosarcoma of the testis. Am J Surg Pathol 2005; 29:420-3

23. Chheda N, Bolegave M, Shet T, Tongaonkar H. Recurrent mullerian adenosarcoma like tumor of seminal vesicle. Indian J Pathol Microbiol 2010; 53:342-4

24. Laurila P, Leivo I, Makisalo H, Ruutu M, Miettinen M. Mullerian adenosarcomalike tumor of the seminal vesicle. A case report with immunohistochemical and ultrastructural observations. Arch Pathol Lab Med 1992; 116:1072-6

25. Mazur MT, Myers JL, Maddox WA. Cystic epithelial-stromal tumor of the seminal vesicle. Am J Surg Pathol 1987; 11:210-7

26. Moch H, Cubilla AL, Humphrey PA, Reuter VE, Ulbright TM. The 2016 WHO Classification of Tumours of the Urinary System and Male Genital Organs. Lyon, France: IARC WHO Classification of Tumours; 2016.

27. Vara AR, Ruzics EP, Moussabeck O, Martin DC. Endometrioid adenosarcoma of the bladder arising from endometriosis. J Urol 1990; 143:813-5 
28. Nezhat CH, Malik S, Osias J, Nezhat F, Nezhat C. Laparoscopic management of 15 patients with infiltrating endometriosis of the bladder and a case of primary intravesical endometrioid adenosarcoma. Fertil Steril 2002; 78:872-5

29. Chen Z, Hong B, Drozd-Borysiuk E, Coffin C, Albritton K. Molecular cytogenetic characterization of a case of Mullerian adenosarcoma. Cancer Genet Cytogenet $2004 ; 148: 129-32$

30. Lee JC, Lu TP, Changou CA, et al. Genomewide copy number analysis of Mullerian adenosarcoma identified chromosomal instability in the aggressive subgroup. Mod Pathol 2016; 29:1070-82

31. Howitt BE, Sholl LM, Dal Cin P, et al. Targeted genomic analysis of Mullerian adenosarcoma. $J$ Pathol 2015; 235:37-49

32. Piscuoglio S, Burke KA, $\mathrm{Ng} \mathrm{CK}$, et al. Uterine adenosarcomas are mesenchymal neoplasms. J Pathol 2016; 238:381-8

33. Blom R, Guerrieri C. Adenosarcoma of the uterus: a clinicopathologic, DNA flow cytometric, p53 and mdm-2 analysis of 11 cases. Int J Gynecol Cancer 1999; 9:3743

34. Varela I, Tarpey P, Raine K, et al. Exome sequencing identifies frequent mutation of the SWI/SNF complex gene PBRM1 in renal carcinoma. Nature 2011; 469:539-42

35. Lee TK, Miyamoto H, Osunkoya AO, et al. Smooth muscle neoplasms of the urinary bladder: a clinicopathologic study of 51 cases. Am J Surg Pathol 2010; 34:502-9

36. Cheng L, Zhang S, Alexander R, et al. Sarcomatoid carcinoma of the urinary bladder: the final common pathway of urothelial carcinoma dedifferentiation. Am J Surg Pathol 2011; 35:e34-46 
37. Young RH. Tumor-like lesions of the urinary bladder. Mod Pathol 2009; 22 Suppl 2:S37-52

38. Young RH, Scully RE. Clear cell adenocarcinoma of the bladder and urethra. A report of three cases and review of the literature. Am J Surg Pathol 1985; 9:816-26

39. Tian W, Latour M, Epstein JI. Endometrial stromal sarcoma involving the urinary bladder: a study of 6 cases. Am J Surg Pathol 2014; 38:982-9

40. Parker RL, Dadmanesh F, Young RH, Clement PB. Polypoid endometriosis: a clinicopathologic analysis of 24 cases and a review of the literature. Am J Surg Pathol 2004; 28:285-97

41. Arend R, Bagaria M, Lewin SN, et al. Long-term outcome and natural history of uterine adenosarcomas. Gynecol Oncol 2010; 119:305-8 


\section{Figure Legends}

Figure 1. Primary urinary bladder müllerian adenosarcoma (hematoxylin and eosin staining). A. Benign-appearing glands are lined by predominantly cuboidal cells with eosinophilic cytoplasm. B. Low-grade müllerian adenosarcoma infiltrating into perivesical fat. C, D. Densely cellular endometrial-type stroma with mild nuclear atypia and increased mitotic figures surrounding benign glands that is often referred to as “cuffing" or "collaring". E. Looser, edematous, and somewhat myoid-appearing stroma. F. Intraglandular, papillary-like projections resembling phyllodes tumor of the breast.

Figure 2. Lymphovascular space invasion. The presence of low-grade müllerian adenocarcinoma within the large vessels of the urinary bladder ( H\&E stain, A: 4x and C: 20x magnifications) highlighted by an immunohistochemical stain for ERG ( $\mathrm{B}, 4 \mathrm{x}$ and $\mathrm{D}$, $20 \mathrm{x})$

Figure 3. Positive immunohistochemical staining within glandular component. A. Pax 8 and B. CK7. Positive immunohistochemical staining within sarcomatous component. C. CD10 D. WT-1 and E. smooth muscle actin. Positive immunohistochemical staining in both morphologic components. F. estrogen receptor G. progesterone receptor and H. CA125

Figure 4. X Chromosome inactivation analysis and virtual karyotypes of Mullerian Adenosarcoma. A-C. Laser microdissection of müllerian adenosarcoma of the urinary bladder. A, tumor before microdissection; B, tumor after microdissection; and C, laser- 
captured tumor cells. D. A clonal pattern in the stromal component that is characterized by a single androgen receptor band after digestion with HhaI restriction endonuclease is demonstrated in this micrograph. For comparison, the epithelial component revealed a random pattern of $\mathrm{X}$ chromosome inactivation. E. The uppermost plot represents the estimated copy number as a $\log 2$ ratio averaged over 30 SNPs; green bars represent heterozygote SNPs; middle bar represents a color-coded Hidden Markov Model (HMM) for copy number (yellow, copy number 2 ; pink, copy number 3 ; aqua, copy number 1 ), and bottom bar is a color-coded HMM for LOH (yellow, no LOH; blue, LOH). This profile shows discrete regions of gain in chromosome $1 \mathrm{q}$ and loss in chromosome $3 \mathrm{p}$. 
A

B

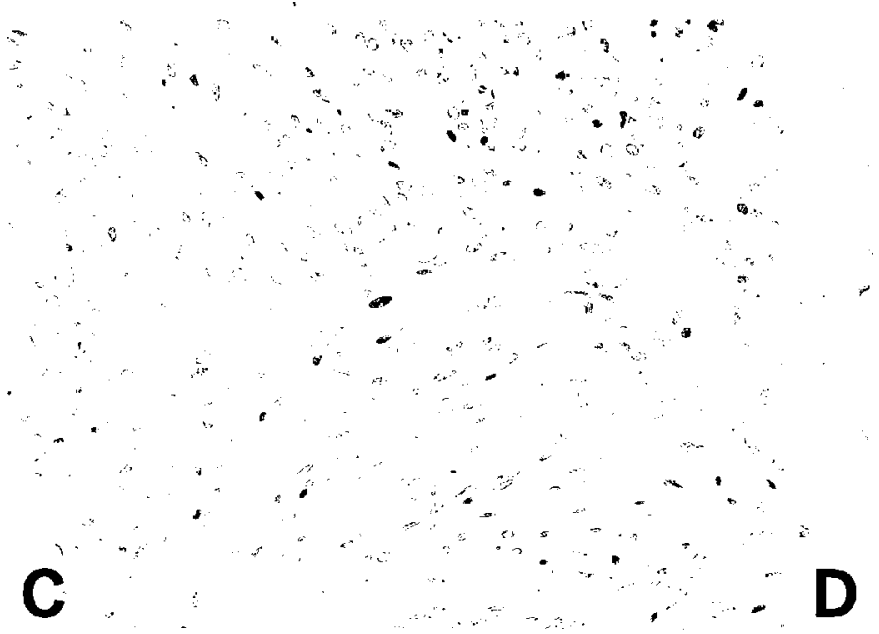

E 
A

B

C

D 


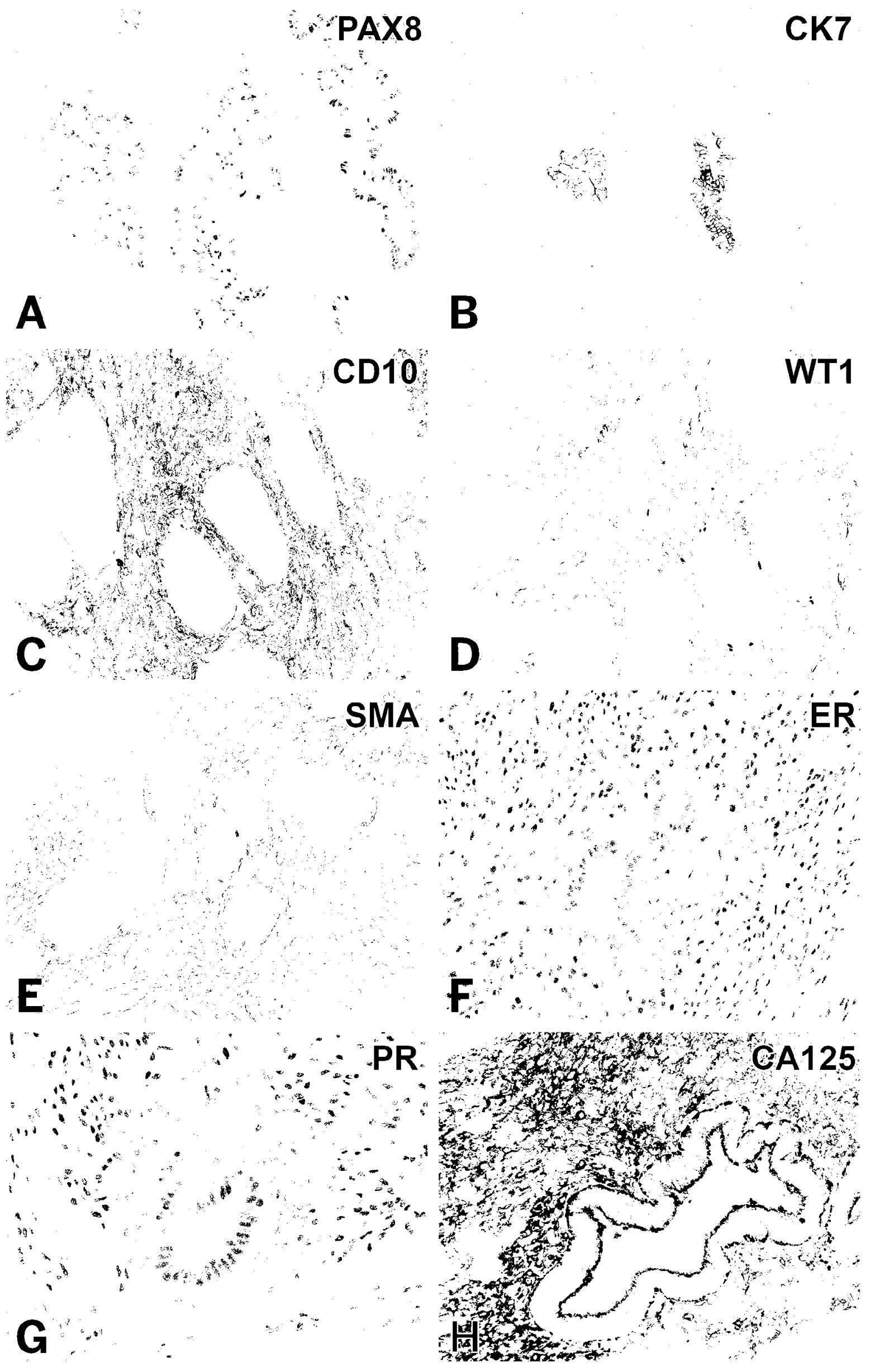


A

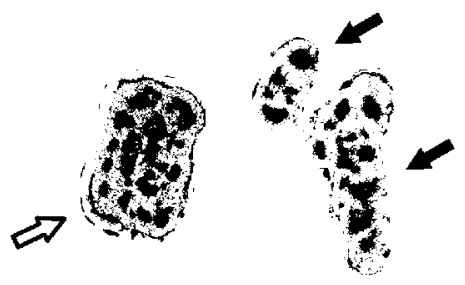

C

Lovir! 1

(1)

1

E
B
D
$\frac{N}{-\quad+} \frac{\text { Sarcoma }}{-} \frac{\text { Epithelial }}{-\quad+}$

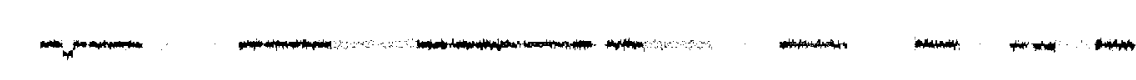

